\title{
The Relationship between Kinesiophobia and Return to Sport after Shoulder Surgery for Recurrent Anterior Instability
}

\author{
Alberto Vascellari ${ }^{1}$ Carlo Ramponi ${ }^{1}$ Davide Venturin ${ }^{1}$ Giulia Ben ${ }^{2}$ Nicolò Coletti ${ }^{3}$
}

${ }^{1}$ Kinè Physiotherapic and Orthopedic Center, San Vendemiano,

Treviso, Italy

Address for correspondence Alberto Vascellari, Kinè Physiotherapic

${ }^{2}$ High Altitude Pediatric Asthma Center, Misurina Pio XII Institute, and Orthopedic Center, Via Venezia 13/q, 31020, San Vendemiano, Belluno, Italy

${ }^{3}$ Department of Orthopaedic and Traumatology, Oderzo Hospital, Oderzo, Treviso, Italy

Joints 2019;7:148-154.

\begin{abstract}
Keywords

- kinesiophobia

- return to sport

- DOSIS scale

- shoulder instability

Purpose To evaluate the relationship between kinesiophobia and patient's return to sport after shoulder stabilization surgery. The hypothesis was that kinesiophobia represents an independent factor correlated to the difference between preinjury and postoperative level of sport.

Methods This study retrospectively evaluated 66 patients (mean age: 35.5 , standard deviation $[S D]=9.9$ years) and at a mean follow-up of $61.1(S D=37.5)$ months after arthroscopic Bankart's repair or open Bristow-Latarjet procedure. Kinesiophobia was assessed with the Tampa Scale for Kinesiophobia (TSK); return to the preinjury sport was assessed by the difference between baseline and postoperative degree of shoulder involvement in sport (D-DOSIS) scale. The Western Ontario Shoulder Instability index (WOSI) was used to evaluate participants' perceptions of shoulder function.

Results TSK showed correlation with D-DOSIS $(\rho=0.505, p<0.001)$ and the WOSI score $(\rho=0.589, p<0.001)$. There was significant difference in TSK and WOSI scores between participants who had and had not returned to their previous level of sport participation ( $p=0.006$, and 0.0001 , respectively).

Conclusion This study demonstrated that kinesiophobia is correlated to the return to sport after shoulder stabilization surgery.

Level of Evidence Level IV, retrospective case series.
\end{abstract}

\section{Introduction}

Glenohumeral instability remains a common and serious concern, especially when it affects athletes and active patients. Shoulder stabilization surgery, whether open or arthroscopic, has proven to be an effective intervention to prevent recurrence while allowing athletes to return to sport activity. ${ }^{1}$ The majority of patients expect good shoulder function and a return to the preinjury level of sport after arthroscopic shoulder surgery. ${ }^{2-4}$ However, a wide-ranging rate of patients may not return to the preinjury sport activity, despite being physically recovered ${ }^{2-11}$ which raises the received

September 12, 2017

accepted

April 18, 2021

published online

June 18, 2021
DOI https://doi.org/ $10.1055 / \mathrm{s}-0041-1730975$. ISSN 2282-4324.

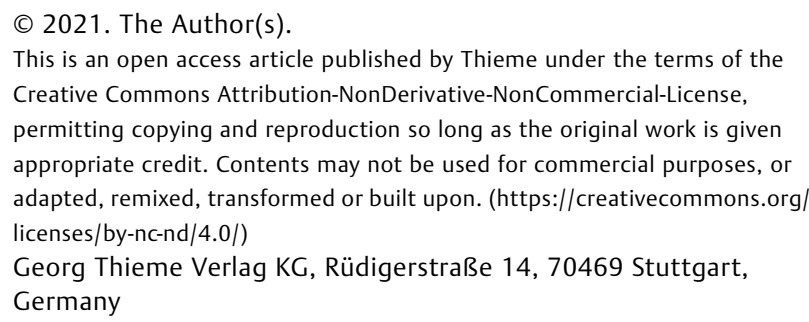


question of what other factors could impact on returning to sports. Recent research has suggested that psychological factors, such as fear of reinjury, low confidence, and selfdetermination may affect athlete's ability to return to the previous level of sport participation. ${ }^{7-13}$ Given that returning to sport is the main criterion by which a successful outcome is judged, investigation of the effect of psychological responses on whether athletes successfully return to sport is important. ${ }^{9}$ Fear of reinjury in athletes, also called kinesiophobia, ${ }^{14}$ is a primary psychosocial construct in the fear-avoidance model. ${ }^{15}$ Athletes who report fear of reinjury are likely to exhibit elevated kinesiophobia, ${ }^{16-19}$ and fear of reinjury can adversely result in both physiological (such as muscular guarding) and psychological changes (such as distraction and lack of trust in the injured site) that can affect rehabilitation outcomes. ${ }^{20}$ Although the effect of kinesiophobia on return to sport has been thoroughly investigated after anterior cruciate ligament reconstruction, 9,11,17,21,22 studies dedicated to patients with shoulder instability remain sparse..$^{3,8,9,13}$

Therefore, the aim of the current study was to analyze the relationship between kinesiophobia and patient's return to sport after shoulder stabilization surgery. The hypothesis was that a relationship exists between kinesiophobia and the difference between preinjury and postoperative level of sport. A secondary hypothesis was that fear of reinjury and perception of shoulder function were significantly different between participants who had and had not returned to their previous level of sport participation.

\section{Methods}

\section{Design}

The study was approved by the Local Hospital Ethics Committee. The study has been performed in accordance with the ethical standards in the 1964 Declaration of Helsinki and has been performed in accordance with relevant regulations of the Italian National Healthcare System.

The study was conducted as a questionnaire-based survey in a series of patients who were affected by recurrent anterior shoulder instability and who underwent an arthroscopic Bankart's repair or an open Bristow-Latarjet procedure. Completion of the online questionnaire used in this study was accepted as implied consent to participate.

\section{Participants}

The digital patient database of the Orthopaedic and Traumatology Department was retrospectively reviewed to identify all of the patients surgically treated for recurrent anterior shoulder instability between January 2005 and December 2015. The inclusion criteria for this study were patients treated by arthroscopic Bankart's repair or open BristowLatarjet procedure, performed at a single hospital by two experienced shoulder surgeons (N.C. and A.V.). Exclusion criteria were concomitant injuries (cuff tear, superior labral anterior-posterior [SLAP] lesion), and epilepsy. A total of 149 patients was considered eligible for the study.

\section{Surgical Techniques and Postoperative Rehabilitation}

The arthroscopic Bankart's stabilization was done with two minimum anchors in all patients and an associated southnorth capsular shift and retensioning was performed in the lateral decubitus position. ${ }^{23}$ Different anchors were used over the course of the study period. The number and type of anchors were dependent on the surgeon's preferences and on the extent of the labral tear. The open Bristow-Latarjet procedure was performed in a similar manner to the technique described by Edwards and Walch. ${ }^{24}$ Two malleolar screws were used in all cases to fix the coracoid to the glenoid in the lying down position and via a horizontal split in the subscapularis muscle. The postoperative regime was the same for both groups. After surgery, the patients were placed in a sling with a pillow spacer for 4 weeks. The sling positioned the upper arm in 90 degrees of elbow flexion, 20 degrees of abduction, and 0 degrees of external rotation at the shoulder. Passive and pain-free motion up to 10 degrees of external rotation and 70 to 80 degrees of internal rotation were allowed immediately after surgery. Pendulum exercises were avoided. After 2 weeks, the patients were allowed to recover progressive abduction up to 90 degrees. From 5 to 8 weeks, patients were allowed to perform passive and active motion up to 0 degrees of external rotation and 90 degrees of abduction, with the support of a physical therapist. Starting from 9 weeks postoperatively, full range of motion (ROM) was allowed. Noncollision sports were allowed starting from 3 to 5 months postoperatively. Collision sports were permitted after 6 months.

\section{Data Collection}

An open-source platform (https://drive.google.com) was configured to collect the responses anonymously. A total of 149 selected patients were contacted by telephone to present the research and to invite them to participate in the study and to fill in online the following self-reported outcome measures: the Degree of Shoulder Involvement in Sport (DOSIS) scale, ${ }^{25}$ and the validated Italian versions of the Tampa Scale for Kinesiophobia (TSK) ${ }^{26}$ and the Western Ontario Shoulder Instability index (WOSI). ${ }^{27}$ The patients were asked to answer the DOSIS score retrospectively by recalling the period of time before the onset of shoulder instability (baseline score) and at follow-up examination (postoperative score). Fifty-one patients were unable to be contacted for reasons such as incorrect contact information and unanswered telephone messages.

\section{Outcome Measures}

The primary outcome was return to the preinjury sport activity, assessed by the difference between baseline and postoperative DOSIS score (D-DOSIS). The predictor variable was kinesiophobia, assessed by the TSK. The secondary predictor variable was patients' perceptions of shoulder function, assessed by the WOSI.

The DOSIS scale is a modified Tegner's activity scale weighted for the shoulder based on the following three parameters: (1) the type of sport, (2) the frequency the sport was played, and (3) the level at which the sport was played. 
Patients then obtain a score from 0 (no sport) to 10 (highdemand sport played by national/international level or professional athlete). ${ }^{25}$ The DOSIS scale has been showed to have an adequate validity and responsiveness for sport-specific shoulder assessment in patients after surgery for anterior instability. $^{28}$

The $\mathrm{TSK}^{26}$ is a widely used questionnaire for assessing pain beliefs and pain-related fear of movement/reinjury in the musculoskeletal field. A 13-item version of the original 17-item self-reported questionnaire TSK was used, with higher scores representing stronger fear-avoidance beliefs in two subscales called Activity Avoidance-TSK1 (a belief that activities causing pain should be avoided) and HarmTSK2 (a belief that pain is a sign of bodily damage).

The WOSI is a disease-specific PROM designed to be used as a primary outcome measure in clinical trials that evaluated treatments for patients with shoulder instability. ${ }^{27}$ The 21-item questionnaire consists of four domains, referring to physical symptoms, sport/recreation/work function, lifestyle function, and emotional function. Originally responses range from no complaints $(0)$ to severe complaints. ${ }^{14}$

\section{Statistical Analysis}

Descriptive statistics were calculated for all variables. Age was dichotomized based on the median age of the cohort. The Kolmogorov-Smirnov test was used to assess the assumption of normality, showing a distribution of the values distant from a normal distribution. A nonparametric analysis of the data was therefore performed. Spearman's rank correlation coefficient $(\rho)$ was used to assess the correlation of kinesiophobia (TSK) with difference between baseline and postoperative level of sport (D-DOSIS), and participants' perceptions of shoulder function (WOSI). The analysis on the correlation of kinesiophobia with the measured outcomes was performed separately for the TSK score and the two TSK subscales. Spearman's coefficient was read as follows: very high correlation for values $>0.9$; high correlation for values between 0.7 and 0.9 ; moderate correlation for values between 0.5 and 0.7 ; low correlation for values between 0.3 and 0.5 ; slight, almost negligible relationship for values $<0.3 .^{29}$ Finally, a multivariate analysis was performed to test whether patient demographic data influenced the patient's return to sport correlation with kinesiophobia or whether kinesiophobia represents an independent factor correlated to the difference between preinjury and postoperative level of sport.

\section{Between-Group Analyses}

TSK score, the two TSK subscales, WOSI score, and the WOSIsport subscore were compared between participants who had or had not returned to their preinjury sport or recreational activity (D-DOSIS $\leq$ or $>0$ ). Pearson's Chi-square and Mann-Whitney $U$-tests were applied for group comparisons. Alpha corrections were made using Benjamini and Hochberg's false discovery rate (FDR) approach. ${ }^{30}$ Unadjusted and adjusted significance values were calculated and presented to provide an indication of the likelihood of both type-I and type-II error rates.

\section{Multivariate Analyses}

A multivariate linear regression analysis was performed to test whether time from surgery to follow-up, age, sex, type of surgery, preinjury activity level, and sport classification according to demand on the upper extremity influenced the D-DOSIS correlation with kinesiophobia, or whether kinesiophobia represented an independent factor correlated to return to the preinjury sport after shoulder stabilization surgery. D-DOSIS was the outcome variable. The analysis included all the variables which were found to be significantly related to the D-DOSIS in the univariate analysis (stepwise forward with an inclusion level of $p \geq 0.25$ ). At this stage age, side, involvement of the dominant arm, number of dislocations before surgery, sport classification according to demand on the upper extremity, and time from surgery to follow-up were excluded from further analysis. Type of surgery has been confounder in prior research ${ }^{31}$ and was retained. The variables that were retained were checked for multicollinearity. A variance inflation factor of $>5$ was used to denote significant multicollinearity. The WOSI-sport score and the two TSK subscales were excluded from further analyses due to significant multicollinearity. The remaining variables were entered into the final model. A $p<0.05$ was considered statistically significant. Data were entered into a Microsoft Excel spreadsheet (Microsoft Corporation, Redmond, Washington) and analyzed using PSPP software (Free Software Foundation, Inc.) for windows.

\section{Results}

Of the 98 patients who responded, 66 patients (67\%) completed the questionnaires and were included in the final analysis for the current study. The mean follow-up time from surgery was 61.1 months (range: 12-156). The demographic data of the cohort are listed in - Table 1.

The majority of participants were active at low level of competition (regional, local) while nine participants were active at a high level of competition (national or international or professional). Regarding DOSIS classification of sports according to demand on the upper extremity, at the time of the onset of shoulder instability participants were most commonly participating in moderate demand sport activity; 24 participants were participating in high demand sport activity.

At follow-up, 31 participants (47.0\%) had returned to their preinjury sport activity (D-DOSIS $\leq 0$ ).

D-DOSIS showed correlation with TSK, TSK1, and TSK2 $(\rho=0.505, \quad p<0.001 ; \quad \rho=0.485, \quad p<0.001 ; \quad \rho=0.402$, $p<0.001$, respectively). The WOSI score showed correlation with TSK, TSK1, and TSK2 $(\rho=0.589, p<0.001 ; \rho=0.619$, $p<0.001 ; \rho=0.484, p<0.001$, respectively).

There were significant differences in psychological and appraisal of shoulder function factors between participants who had and had not returned to their previous level of sport participation. Participants who had returned to their previous activity reported higher perceptions of shoulder function (WOSI) and lower fear of reinjury (TSK). They also reported better shoulder function in sport and recreational activities (WOSI-Sport; - Table 2 ). 
Table 1 Demographic data for study cohorts

\begin{tabular}{|c|c|c|c|c|}
\hline \multirow[b]{3}{*}{ Variable } & \multirow{3}{*}{$\begin{array}{l}\text { Overall } \\
(n=66)\end{array}$} & \multicolumn{2}{|c|}{ Difference between baseline } & \multirow[b]{3}{*}{$p$-Value } \\
\hline & & \multicolumn{2}{|c|}{ and postoperative DOSIS } & \\
\hline & & $\leq 0(n=31)$ & $>0(n=35)$ & \\
\hline $\begin{array}{l}\text { Age at surgery }(y) \\
\text { Mean (SD) }\end{array}$ & $35.5(9.9)$ & $35.2(10.2)$ & $35.7(9.8)$ & 0.73 \\
\hline $18-35$ & & $17(54.8 \%)$ & $18(51.4 \%)$ & 0.78 \\
\hline $36-50$ & & $14(45.2 \%)$ & $17(48.6 \%)$ & \\
\hline Sex & & & & 0.15 \\
\hline Male & $50(75.8 \%)$ & $26(83.9 \%)$ & $24(68.6 \%)$ & \\
\hline Female & $16(24.2 \%)$ & $5(16.1 \%)$ & $11(31.4 \%)$ & \\
\hline Side & & & & 0.89 \\
\hline Right & $41(62.1 \%)$ & $19(61.3 \%)$ & $22(62.9 \%)$ & \\
\hline Left & $25(37.9 \%)$ & $12(38.7 \%)$ & $13(37.1 \%)$ & \\
\hline Surgery on dominant shoulder & & & & 0.51 \\
\hline Yes & $41(62.1 \%)$ & $17(54.8 \%)$ & $22(62.9 \%)$ & \\
\hline No & $25(37.9 \%)$ & $14(45.2 \%)$ & $13(37.1 \%)$ & \\
\hline $\begin{array}{l}\text { Number of shoulder dislocations before surgery } \\
\text { Mean (SD) }\end{array}$ & $5.5(5.0)$ & $5.3(5.0)$ & $5.7(5.0)$ & 0.83 \\
\hline Preinjury activity level & & & & 0.08 \\
\hline High level of competition & $9(13.6 \%)$ & $3(9.7 \%)$ & $6(17.1 \%)$ & \\
\hline Low level of competition & $38(57.6 \%)$ & $15(48.4 \%)$ & $23(65.7 \%)$ & \\
\hline Recreational & $19(28.8 \%)$ & $13(41.9 \%)$ & $6(17.1 \%)$ & \\
\hline Preinjury sport classification & & & & 0.31 \\
\hline High demand & $24(36.4 \%)$ & $11(35.5 \%)$ & $13(37.1 \%)$ & \\
\hline Moderate demand & $28(42.4 \%)$ & $11(35.5 \%)$ & $17(48.6 \%)$ & \\
\hline No or minimal demand & $14(21.2 \%)$ & $9(29.0 \%)$ & $5(14.3 \%)$ & \\
\hline Type of surgery & & & & 0.39 \\
\hline Arthroscopic Bankart's repair & $52(78.8 \%)$ & $23(74.2 \%)$ & $29(82.9 \%)$ & \\
\hline Open Bristow-Latarjet procedure & $14(21.2 \%)$ & $8(25.8 \%)$ & $6(17.1 \%)$ & \\
\hline $\begin{array}{l}\text { Time from surgery to follow-up (mo) } \\
\text { Mean (SD) }\end{array}$ & $61.1(37.5)$ & $58.0(38.0)$ & $64.2(37.3)$ & 0.46 \\
\hline
\end{tabular}

Abbreviations: DOSIS, degree of shoulder involvement in sport; SD, standard deviation.

Note: Demographic data were compared between participants who had or had not returned to their preinjury sport activity (difference between baseline and postoperative DOSIS $\leq$ or $>0$ ).

Table 2 Comparisons of fear of reinjury and participants' perceptions of shoulder function between participants who had and had not returned to their preinjury level of sport participation

\begin{tabular}{|l|l|l|l|l|l|l|}
\hline & & \multicolumn{2}{l|}{ Difference between baseline } & & \\
\hline & Overall & \multicolumn{2}{|l|}{ and postoperative DOSIS } & Mean difference & & FDR corrected \\
\hline Variable & Mean (SD) & $\leq \mathbf{0}(\boldsymbol{n}=\mathbf{3 1})$ & $>\mathbf{0}(\boldsymbol{n}=\mathbf{3 5})$ & {$[\mathbf{9 5 \%} \mathrm{CI}]$} & $\boldsymbol{p}$-Value & significance, $\boldsymbol{q}$ \\
\hline TSK & $27.0(9.2)$ & $23.4(7.4)$ & $30.1(9.6)$ & $-6.7[-6.0$ to -7.4$]$ & 0.006 & 0.0075 \\
\hline TSK-I & $11.5(4.5)$ & $9.52(3.39)$ & $13.2(4.7)$ & $-3.7[-3.3$ to -4.0$]$ & 0.004 & 0.0066 \\
\hline TSK-II & $15.5(5.3)$ & $13.9(4.8)$ & $16.9(5.3)$ & $-3.0[-2.6$ to -3.4$]$ & 0.04 & 0.04 \\
\hline WOSI & $3.5(2.5)$ & $1.9(1.8)$ & $4.5(2.7)$ & $2.6[2.4$ to 2.8$]$ & 0.0001 & 0.00025 \\
\hline WOSI-Sport & $3.1(3.2)$ & $1.5(2.3)$ & $4.6(3.3)$ & $3.1[2.9$ to 3.3] & 0.0001 & 0.00025 \\
\hline
\end{tabular}

Abbreviations: $\mathrm{Cl}$, confidence interval; DOSIS, degree of shoulder involvement in sport; FDR, false discovery rate; SD, standard deviation; TSK, the Tampa Scale for Kinesiophobia; WOSI, Western Ontario shoulder instability index. 
Table 3 Regression model of the relationship between returning to the preinjury sport activity and fear of reinjury participants' perceptions of shoulder function and demographic factors

\begin{tabular}{|l|l|l|l|l|}
\hline & Coefficient & Standard error & Stat t & $p$ (95\% Cl) \\
\hline WOSI & 0.228 & 0.202 & 1.130 & 0.263 (-0.176 to 0.632) \\
\hline TSK & 0.146 & 0.063 & 2.299 & 0.025 (0.019 to 0.273) \\
\hline Sex & -1.377 & 0.913 & -1.508 & 0.137 (-3.206 to 0.452) \\
\hline Level of sport & 1.149 & 0.577 & 1.992 & 0.051 (-0.006 to 2.304) \\
\hline Type of surgery & 0.579 & 0.939 & 0.617 & 0.540 (-1.301 to 2.460) \\
\hline
\end{tabular}

Abbreviations: Cl, confidence interval; DOSIS, degree of shoulder involvement in sport; TSK, the Tampa Scale for Kinesiophobia; WOSI, Western Ontario shoulder instability index.

The multivariate analysis included TSK and WOSI scores, sex, type of surgery, and preinjury activity level. The final model $\left(\mathrm{F}=6.799, p<0.001, R^{2}=0.374\right)$ confirmed that TSK score represented an independent factor correlated to returning to the preinjury activity, while there was no significant correlation with preinjury activity level (-Table 3). There were no significant correlation between D-DOSIS and WOSI, sex, and type of surgery (- Table 3 ).

\section{Discussion}

The most important finding of the present study was that kinesiophobia is correlated to returning to the preinjury sport activity after shoulder stabilization surgery. Participants who had returned to their preinjury sport activity reported lower kinesiophobia, compared with participants who had not returned to their preinjury sport activity. Further, those who had returned to their preinjury sport activity were more satisfied with their current shoulder function in sport activities. These findings add to a growing body of the literature suggesting that fear of reinjury seems to be a key issue in sports injury rehabilitation and returning to the preinjury activity level and satisfaction. ${ }^{9,18,32,33}$ Kinesiophobia has been reported to be related to reduced self-report function, low level of physical activity, and has been identified as a key element in a patient's psychological readiness to return to sport after anterior cruciate ligament (ACL) reconstruction, tibia and fibula fracture, elbow injuries, and the Achilles tendon rupture. ${ }^{19,21,34-37}$ In a recent study, exploring the specific factors influencing a patient's decision to return to sport after shoulder stabilization surgery, the fear of redislocation was found to be the most prevalent patient-derived theme influencing a negative decision to return to sports. ${ }^{13}$ Many patients recalled their initial feeling of apprehension before the injury and expressed a similar feeling on their initial return to sports. While those who decided to return to sports overcame their initial fear of reinjury, many who did not return to sports still feared redislocations. Gerometta et $\mathrm{al}^{38}$ in a retrospective study evaluating return to sports after arthroscopic Bankart's stabilization in athletes, introduced the subjective concept of avoidance. Of the 46 athletes surveyed, $37 \%$ still experienced voluntary avoidance of certain movements postoperatively. Although this does not necessarily correlate with the ability of a patient to return to sport, it likely relates to a degree of kinesiophobia throughout recovery.

Returning to the preinjury sport activity was associated with higher perceptions of shoulder function. Gerometta et $\mathrm{al}^{38}$ reported that the WOSI score was significantly worse in patients who did not return to their athletic activities, in case the athlete had to change her/his sport and in case of return to sports on an inferior level. Conversely, Tjong et al reported that the decision of a patient not to return to sport after arthroscopic shoulder stabilization was not attributable to a perceived lack of shoulder function. ${ }^{13}$

The results of this study revealed that only $47 \%$ of patients returned to their preinjury level of sport. These results are comparable to the current literature with return to sports of $44 \%$ after arthroscopic shoulder stabilization ${ }^{13}$ and $63.8 \%$ after Latarjet-Bristow procedure. ${ }^{39}$ Other studies reported much higher values; in their case series of arthroscopic shoulder stabilization, Ozturk et $\mathrm{al}^{7}$ reported a return to the same preinjury level of sport in $75 \%$ of patients. Gerometta et $\mathrm{al}^{38}$ reported that $95.7 \%$ of patients returned to the same level of play after arthroscopic Bankart's stabilization. One possible explanation for this discrepancy could be attributed to this study's strict definition of "return to sport" which is commonly ill defined in other sources. In our study, a new scale (DOSIS scale) has been used to classify patients on the basis of their sport activity based on the specific involvement of the shoulder in that sport.

\section{Limitations}

This study has several limitations that need to be discussed. First, this was a retrospective study with a wide follow-up time range. Thus, the recall bias may differs between participants that had their surgery 1 and 10 years before evaluation. Second, a power analysis was not performed. Due to our small sample size, a subgroup analysis could not be conducted, and generalizations to other samples with shoulder instability may be affected and therefore the results need to be confirmed in future studies. Another limitation is that the data were collected in a retrospective manner in part, since we asked patients to recall their sport activity levels (baseline and preoperative DOSIS). The relevancy of this limitation was tested in the original validation study of the DOSIS scale by comparing the test-retest reliability of the DOSIS scale measured retrospectively and the DOSIS scale measured at follow-up (postoperative DOSIS). No significant differences 
were found, suggesting that the DOSIS scale is reliable even when it is measured retrospectively. ${ }^{25}$ Another limitation is the minimum follow-up of 1 year. This study, however, was focused on the return to sport which generally does not require a lengthy follow-up to be assessed. Participation bias also exists because only $67 \%$ of potential participants responded to the battery of patient-reported outcomes. It is possible that, given the number of patient-reported outcomes included in the battery, some participants were deterred from completing it. Patients who agree to be interviewed may have had a different rate of return to the preinjury sport activity, responses to the psychological outcomes, and perceptions of shoulder function than those who declined. However, this response rate is above the 50\% minimum suggested to reduce bias. ${ }^{11,40}$

The clinical relevance of this study is that functional outcome scores alone cannot predict the propensity to return to sport after shoulder stabilization surgery, and a stronger emphasis on addressing psychological readiness to return to activity in postoperative rehabilitation programs could be warranted to help patients reporting a lack of confidence in the shoulder or fear of sustaining a new injury. Future investigations may strengthen the results obtained in this study and investigate the potential effects of kinesiophobia-reducing interventions, such as cognitive-behavioral therapy, to identify patients at risk and overcome barriers to full recovery after shoulder stabilization surgery.

\section{Conclusion}

This study demonstrated that kinesiophobia is correlated to the return to sport after shoulder stabilization surgery, and that patients who return to preinjury sport activity level report lower levels of kinesiophobia than patients who do not return. Further, patients who return to preinjury sport activity are more satisfied with their current shoulder function in sport activities.

Note

All patients gave their informed consent upon receiving complete information on the study. Completion of the online questionnaire used in this study was accepted as implied consent to participate.

\section{Ethical Approval}

The study was approved by the Local Hospital Ethics Committee (Prot. 78695).

Funding

None.

\section{Conflict of Interest}

None declared.

\section{Acknowledgments}

The authors are grateful to Trina Lat, The University of Queensland, for English editing.

\section{References}

1 Mohtadi NG, Chan DS, Hollinshead RM, et al. A randomized clinical trial comparing open and arthroscopic stabilization for recurrent traumatic anterior shoulder instability: two-year follow-up with disease-specific quality-of-life outcomes. J Bone Joint Surg Am 2014;96(05):353-360

2 Bliven KCH, Parr GP. Outcomes of the latarjet procedure compared with bankart repair for recurrent traumatic anterior shoulder instability. J Athl Train 2018;53(02):181-183

3 Donohue MA, Owens BD, Dickens JF. Return to play following anterior shoulder dislocation and stabilization surgery. Clin Sports Med 2016;35(04):545-561

4 Warth RJ, Briggs KK, Dornan GJ, Horan MP, Millett PJ. Patient expectations before arthroscopic shoulder surgery: correlation with patients' reasons for seeking treatment. J Shoulder Elbow Surg 2013;22(12):1676-1681

5 Cohen SB, Sheridan S, Ciccotti MG. Return to sports for professional baseball players after surgery of the shoulder or elbow. Sports Health 2011;3(01):105-111

6 Fedoriw WW, Ramkumar P, McCulloch PC, Lintner DM. Return to play after treatment of superior labral tears in professional baseball players. Am J Sports Med 2014;42(05):1155-1160

7 Ozturk BY, Maak TG, Fabricant P, et al. Return to sports after arthroscopic anterior stabilization in patients aged younger than 25 years. Arthroscopy 2013;29(12):1922-1931

8 Watson S, Allen B, Grant JA. A clinical review of return-to-play considerations after anterior shoulder dislocation. Sports Health 2016;8(04):336-341

9 Ialenti MN, Mulvihill JD, Feinstein M, Zhang AL, Feeley BT. Return to play following shoulder stabilization: a systematic review and metaanalysis. Orthop J Sports Med 2017;5(09):2325967117726055

10 Ardern CL, Taylor NF, Feller JA, Webster KE. A systematic review of the psychological factors associated with returning to sport following injury. Br J Sports Med 2013;47(17):1120-1126

11 Ardern CL, Österberg A, Tagesson S, Gauffin H, Webster KE, Kvist J. The impact of psychological readiness to return to sport and recreational activities after anterior cruciate ligament reconstruction. Br J Sports Med 2014;48(22):1613-1619

12 Kontos A, Feltz D, Malina R. The development of the Risk of Injury in Sports Scale (RISSc). J Sport Exerc Psychol 2000;22:S10

13 Tjong VK, Devitt BM, Murnaghan ML, Ogilvie-Harris DJ, Theodoropoulos JS. A qualitative investigation of return to sport after arthroscopic bankart repair: beyond stability. Am J Sports Med 2015;43(08):2005-2011

14 Kori S, Miller R, Todd D. Kinesiophobia: a new view of chronic pain behavior. Pain Manage 1990;3:35-43

15 Vlaeyen JWS, Linton SJ. Fear-avoidance and its consequences in chronic musculoskeletal pain: a state of the art. Pain 2000;85(03): 317-332

16 Ardern CL, Webster KE, Taylor NF, Feller JA. Return to sport following anterior cruciate ligament reconstruction surgery: a systematic review and meta-analysis of the state of play. $\mathrm{Br} \mathrm{J}$ Sports Med 2011;45(07):596-606

17 Flanigan DC, Everhart JS, Pedroza A, Smith T, Kaeding CC. Fear of reinjury (kinesiophobia) and persistent knee symptoms are common factors for lack of return to sport after anterior cruciate ligament reconstruction. Arthroscopy 2013;29(08):1322-1329

18 Kvist J, Ek A, Sporrstedt K, Good L. Fear of re-injury: a hindrance for returning to sports after anterior cruciate ligament reconstruction. Knee Surg Sports Traumatol Arthrosc 2005;13(05): 393-397

19 Lentz TA, Tillman SM, Indelicato PA, Moser MW, George SZ, Chmielewski TL. Factors associated with function after anterior cruciate ligament reconstruction. Sports Health 2009;1(01): 47-53

20 Hsu CJ, Meierbachtol A, George SZ, Chmielewski TL. Fear of reinjury in athletes. Sports Health 2017;9(02):162-167 
21 Ardern CL, Österberg A, Sonesson S, Gauffin H, Webster KE, Kvist J. Satisfaction with knee function after primary anterior cruciate ligament reconstruction is associated with self efficacy, quality of life, and returning to the preinjury physical activity. Arthroscopy 2016;32(08):1631-1638

22 Tjong VK, Murnaghan ML, Nyhof-Young JM, Ogilvie-Harris DJ. A qualitative investigation of the decision to return to sport after anterior cruciate ligament reconstruction: to play or not to play. Am J Sports Med 2014;42(02):336-342

23 Cole BJ, Romeo AA. Arthroscopic shoulder stabilization with suture anchors: technique, technology, and pitfalls. Clin Orthop Relat Res $2001 ;(390): 17-30$

24 Edwards TB, Walch G. The Latarjet procedure for recurrent anterior shoulder instability: rationale and technique. Oper Tech Sports Med 2002;10:25-32

25 Blonna D, Bellato E, Bonasia DE, et al. Design and Testing of the Degree of Shoulder Involvement in Sports (DOSIS) Scale. Am J Sports Med 2015;43(10):2423-2430

26 Monticone M, Giorgi I, Baiardi P, Barbieri M, Rocca B, Bonezzi C. Development of the Italian version of the Tampa Scale of Kinesiophobia (TSK-I): cross-cultural adaptation, factor analysis, reliability, and validity. Spine 2010;35(12):1241-1246

27 Cacchio A, Paoloni M, Griffin SH, et al. Cross-cultural adaptation and measurement properties of an Italian version of the Western Ontario Shoulder Instability Index (WOSI). J Orthop Sports Phys Ther 2012;42(06):559-567

28 Vascellari A, Ramponi C, Venturin D, Ben G, Blonna D, Coletti NSIGASCOT Sport Committee. The Degree of Shoulder Involvement in Sports (DOSIS) scale is a valid and responsive instrumentation for shoulder assessment in patients after surgery for anterior instability. Knee Surg Sports Traumatol Arthrosc 2018; 26(01):195-202

29 Hinkle DE, Wiersma W, Jurs SG. Applied Statistics for the Behavioral Sciences. 5th ed. Boston, MA: Houghton Mifflin; 2003

30 Benjamini Y, Hochberg Y. Controlling the false discovery rate: a practical and powerful approach to multiple testing. J R Stat Soc B 1995;57:289-300
31 Blonna D, Bellato E, Caranzano F, Assom M, Rossi R, Castoldi F. Arthroscopic Bankart repair versus open Bristow-Latarjet for shoulder instability: a matched-pair multicenter study focused on return to sport. Am J Sports Med 2016;44(12):3198-3205

32 Clement D, Arvinen-Barrow M, Fetty T. Psychosocial responses during different phases of sport-injury rehabilitation: a qualitative study. J Athl Train 2015;50(01):95-104

33 Podlog L, Eklund RC. A longitudinal investigation of competitive athletes' return to sport following serious injury. J Appl Sport Psychol 2006;18:44-68

34 Feigenbaum LA, Baraga M, Kaplan LD, et al. Return to sport following surgery for a complicated tibia and fibula fracture in a collegiate women's soccer player with a low level of kinesiophobia. Int J Sports Phys Ther 2015;10(01):95-103

35 George SZ, Lentz TA, Zeppieri G, Lee D, Chmielewski TL. Analysis of shortened versions of the Tampa scale for kinesiophobia and pain catastrophizing scale for patients after anterior cruciate ligament reconstruction. Clin J Pain 2012;28(01):73-80

36 Olsson N, Karlsson J, Eriksson BI, Brorsson A, Lundberg M, Silbernagel KG. Ability to perform a single heel-rise is significantly related to patient-reported outcome after Achilles tendon rupture. Scand J Med Sci Sports 2014;24(01):152-158

37 Prugh J, Zeppieri G Jr., George SZ. Impact of psychosocial factors, pain, and functional limitations on throwing athletes who return to sport following elbow injuries: a case series. Physiother Theory Pract 2012;28(08):633-640

38 Gerometta A, Rosso C, Klouche S, Hardy P. Arthroscopic Bankart shoulder stabilization in athletes: return to sports and functional outcomes. Knee Surg Sports Traumatol Arthrosc 2016;24(06): 1877-1883

39 Beranger JS, Klouche S, Bauer T, Demoures T, Hardy P. Anterior shoulder stabilization by Bristow-Latarjet procedure in athletes: return-to-sport and functional outcomes at minimum 2year follow-up. Eur J Orthop Surg Traumatol 2016;26(03): 277-282

40 Rea LM, Parker RA. Designing and Conducting Survey Research: A Comprehensive Guide. San Francisco, CA: Jossey-Bass; 1997 